\title{
Intraoperative Periprosthetic Fractures in Total Hip Arthroplasty in Patients With Sickle Cell Disease at King Fahad Hospital Hofuf: A Cross- Sectional Study
}

Mohammad K. Alsaleem ${ }^{1}$, Hassan A. Alalwan ${ }^{1}$, Abdullah M. Alkhars ${ }^{2}$, Abdullah H. Al Huwaiyshil ${ }^{2}$, Wejdan M. Alamri ${ }^{1}$

1. Orthopedics, King Fahad Hospital Hofuf, Al-Ahsa, SAU 2. Orthopedics, King Faisal University, Al-Ahsa, SAU

Corresponding author: Abdullah M. Alkhars, alkhars123@hotmail.com

\section{Abstract}

\section{Background}

Patients with avascular necrosis related to sickle cell disease (SCD) can be severely disabled by the severe degenerative changes of their hip. Total hip arthroplasty (THA) remains the only surgical option for some of these patients. Total hip arthroplasty can be a challenging procedure, and SCD patients demonstrate high percentages of medical, intraoperative, and postoperative complications and implant failure. Furthermore, the need for THA following avascular necrosis in the Eastern Province of Saudi Arabia is high, and the subsequent risk of periprosthetic fracture is prevalent. Therefore, it is crucial to conduct such a study.

\section{Aim of the study}

This cross-sectional retrospective study aimed to assess the prevalence and associated risk factors for periprosthetic fractures during total hip arthroplasty in sickle cell disease patients at King Fahad Hospital Hofuf, Saudi Arabia.

\section{Methods}

We collected the data of all SCD patients who had undergone THA during the study period, January 2015 to September 2020. Forty-nine SCD patients who had undergone THA during the study period were included. Patients who had undergone hip hemiarthroplasty, postoperative fractures, or had an indication of THA other than avascular necrosis were excluded. Surgeon factors, assistant factors, and surgical technique were also excluded. We then analyzed the data according to gender, age, BMI, American Society of Anesthesiologists classification, implant fixation type, avascular necrosis stage, proximal femoral morphology, Vancouver classification type, sickle cell type, preoperative hemoglobin $(\mathrm{Hb})$ level, and the risk of periprosthetic fractures. Descriptive statistics were presented using frequency and percentages for categorical variables, and continuous variables were summarized using means \pm standard deviations.

Review began 10/24/2020 Review ended 11/08/2020 Published 11/09/2020

\section{() Copyright 2020}

Alsaleem et al. This is an open access article distributed under the terms of the Creative Commons Attribution License CC-BY 4.0., which permits unrestricted use, distribution, and reproduction in any medium, provided the original author and source are credited.
Independent t-tests and chi-square tests were used to test for associations between categorical variables. At 0.05 , the significance level was set.

\section{Results}

Of the patients, $32.7 \%$ were male and $67.3 \%$ were female. $32.7 \%$ of the patients had advanced degenerative changes due to avascular necrosis. Among the patients, $20.4 \%$ had an intraoperative periprosthetic femoral fracture, 90\% had a Vancouver classification class A, and 10\% had a Vancouver classification class B1. According to Dorr classification, $75.5 \%$ were classified as Dorr A and 24.5\% as Dorr B. Of the patients, 48 had an uncemented implant, and only 1 had cemented. The mean perioperative $\mathrm{Hb}$ was $9.02+2.02$, with a minimum of 6 and a maximum of 14 . No significant associations were found between the incidence of intraoperative femoral fracture and the demographic variables and the operative profile characteristics. However, a significantly higher rate of fracture was observed in patients operated on the right side compared to patients operated on the left side.

\section{Conclusion}

The prevalence of periprosthetic intraoperative fracture among SCD patients at King Fahad Hospital Hofuf was $20.4 \%$ during the study period. Even with adequate perioperative management, orthopedic surgeons must be prepared to deal with high rates of intraoperative fracture. No significant association was found between the incidence of intraoperative femoral fracture in SCD patients and the demographic variables and the operative profiles. However, a significantly higher rate of fracture was observed in patients operated on the right side compared to patients operated on the left side. 
Keywords: total hip arthroplasty: tha, avascular necrosis: avn, american society of anesthesiologist: asa, hemoglobin: hb, sickle cell disease: scd

\section{Introduction}

Total hip arthroplasty (THA) is a saving procedure used to relieve pain and functional disability associated with many hip problems, most commonly, primary hip osteoarthritis, posttraumatic arthritis, and avascular necrosis in sickle cell disease patients [1-6]. Sickle cell disease is endemic in Saudi Arabia due to the high percentage $(57.7 \%$ ) of consanguineous marriages, rising to more than $80 \%$ in some rural areas $[7,8]$. Sickle cell disease is most commonly found in the Eastern Province of Saudi Arabia, with a prevalence of 145 cases/10,000 population, which increases the risk of avascular necrosis in these patients $[7,8]$.

Avascular necrosis, a chronic condition that can lead to deterioration of the hip joint and degradation of bone tissue due to a lack of blood supply [9]. The Steinberg classification system includes seven stages, from 0 to VI, according to the extent of involvement and disease progression [9]. At stage III and more advanced stages, THA is required [9].

One of the main complications of THA for sickle cell disease (SCD) patients is periprosthetic hip fracture [16]. The incidence of fracture is affected by gender, age, race, BMI, implant fixation type, proximal femoral morphology, sickle cell genotype, and hemoglobin ( $\mathrm{Hb}$ ) level [7-12]. The Vancouver classification system classifies periprosthetic hip fracture according to the location, condition of the femoral implant, and value of surrounding femoral bone stock [13].

A systemic review and meta-analysis undertaken in 2014 [14] revealed a high incidence of periprosthetic fractures after THA in females older than 80 years. Some studies have found that the incidence of periprosthetic fracture is higher in whites with low BMIs [1-5]. Proximal femoral morphology can be radiologically classified before reconstruction according to the Dorr classification $[15,16]$. Dorr type $C$ is more commonly associated with periprosthetic hip fracture than types A and B $[15,16]$. In addition, many studies have found that uncemented implant fixation is associated with more pain and risk of fracture compared to cemented implant fixation [17,18]. Furthermore, a 2019 meta-analysis [19] revealed a low incidence of periprosthetic fracture in patients treated with cemented implant fixation. A history of SCD and an $\mathrm{Hb}$ level $<6 \mathrm{~g} / \mathrm{dl}$ are associated with a greater risk of periprosthetic fracture [11].

In the Eastern Province, particularly in Al Hasa city, many sickle cell patients require THA following avascular necrosis, and the subsequent risk of periprosthetic fracture is high $[7,8]$. Moreover, periprosthetic fracture largely affects the prognosis of patients who have undergone THA and subsequently increases hospitalization rates threefold [1-6], which can lead to further complications and may result in death.

Therefore, this study was conducted at King Fahad Hospital Hofuf to assess the prevalence and associated risk factors of developing periprosthetic hip fracture in sickle cell disease patients.

\section{Materials And Methods \\ Study area, sampling, and data collection}

A cross-sectional retrospective study was conducted to examine whether gender, age, BMI, American Society of Anesthesiologists (ASA) classification, implant fixation type, avascular necrosis stage, proximal femoral morphology, Vancouver classification type, sickle cell type, and preoperative Hb level were associated with a greater risk of periprosthetic fractures in sickle cell patients treated at King Fahad Hospital Hofuf with THA during the study period, January 2015 to September 2020.

The examined factors were designed and developed through a literature review of periprosthetic intraoperative fractures, and validity was obtained through a review process with experts in the field. Then, the data were collected from the King Fahad Hospital Hofuf database by two orthopedic residents, who had received prior training for this task after obtaining ethical approval.

Forty-nine SCD patients who had undergone THA during the study period were included. Patients who had undergone hip hemiarthroplasty, postoperative fractures, or had an indication of THA other than avascular necrosis (i.e., osteoarthritis, rheumatoid arthritis, traumatic fracture. etc.) were excluded. Surgeon factors, assistant factors, and surgical technique were also excluded.

\section{Study comparison and intervention}

We aimed to determine if gender and age were associated with greater risk. The patients' ages were grouped as follows: young: less than 18 years old; adult: 18-65; and elderly: $65+$. To determine if BMI was associated with greater risk, we used the following BMI categories: underweight $=<18.5$, healthy weight range $=18.5$ 24.9 , overweight $=25-29.9$, class 1 obesity $=30$ to $<35$, class 2 obesity $=35$ to $<40$, and class 3 obesity $=\geqslant 40$. To determines which ASA classification had more risk, we used the flowing categories: ASA 1: a normal healthy patient, ASA 2: a patient with mild systemic disease, ASA 3: a patient with a serious, non-life- 
threatening systemic disease, ASA 4: a patient with a serious, life-threatening systemic disease, ASA 5: a morbid patient not expected to survive without surgery, and ASA 6: a brain-dead patient [20]. The preoperative $\mathrm{Hb}$ levels were classified into 6-9 g/dl, 10-12 g/dl, and >13 g/dl.

Implant fixation was radiologically categorized as cemented or uncemented. Avascular necrosis was radiologically classified by the Steinberg classification system into seven stages: (stage 0) normal or nondiagnostic radiographs, MRI, and bone scan of the at-risk hip; (stage I) normal radiograph, abnormal bone scan and/or MRI; (stage II) cystic and sclerotic radiographic changes; (stage III) subchondral lucency or crescent sign; (stage IV) femoral head flattening; (stage V) narrowing of joint space with or without acetabular involvement; and (stage VI) advanced degenerative changes [21].

Proximal femoral morphology was radiologically classified according to the Dorr classification: (type A) exhibited thick cortices that begin at the distal end of the lesser trochanter and thicken quickly, which lead to the development of a funnel shape and a narrow diaphysal canal; (type B) proximal bone loss and widening of the diaphysal canal; and (type C) substantial loss of cortical thickness resulting in a much wider intramedullary canal and blurred appearance of the cortices of the bone [22].

Fractures of the femur adjacent to the femoral component of a THA were radiographically described using the Vancouver classification system: Type A fractures occur in the trochanteric region and involve either the greater trochanter or the lesser trochanter, and Type B fractures occur around or just distal to the stem of the femoral component, and on the basis of the implant's stability and the surrounding bone stock, they are subclassified [23]. Fractures of type B1 occur around a stable implant. Fractures of type B2 happen around a loose implant with sufficient bone stock. Fractures of type B3 happen around a loose implant with low bone stock. Fractures of Type C occur well distal to a stable femoral element [23].

\section{Statistical analysis}

After data collecting using Microsoft Excel (Microsoft Corporation, Redmond, USA), the data were exported to Statistical Packages for Social Sciences, Version 23 (IBM Corp, Armonk, USA), for analysis. Descriptive statistics were presented using frequency and percentages for categorical variables, and continuous variables were summarized using means \pm standard deviations. The association of periprosthetic fractures with gender, age, BMI, ASA classification, implant fixation type, avascular necrosis stage, proximal femoral morphology, Vancouver classification type, sickle cell type, and preoperative Hb level was tested using an independent $t$-test. A chi-square test was used to test for associations between categorical variables. The level of significance was set at 0.05 .

\section{Results}

A total of 49 sickle cell patients who had undergone THA during the study period were included. In Table 1, the demographic profile of patients is provided. Of the patients, 16 (32.7\%) were male and $33(67.3 \%)$ were female. For the category of BMI, 3 (6.1\%) of the patients were underweight, 26 (43.1\%) had a normal BMI, 13 (26.5\%) were overweight, and $7(14.3 \%)$ had class 1 obesity. The mean age of the patients was $43.43+11.69$ years; the minimum age was 23 , and the maximum age was 68 . 


\section{Cureus}

Demographic Characteristics

Gender

Male

Female

BMI

Underweight

Normal BMI

Overweight

Class 1 Obesity

Age (mean, SD)

Mean

41.96

Standard Deviation

Minimum

Maximum n

$\%$

16

32.70

33

67.30

3

6.10

53.10

13

26.50

7

14.30

TABLE 1: Demographic profile of the patients $(n=49)$

Table 2 shows the operation profile of the patients. The ASA classification for the patients was as following, 3 (6.1\%) were categorized into class 1,28 (57.1\%) were categorized into class 2 and 18 (36.7\%) were categorized as class 3 . As for the operated side, 20 (40.8\%) of the patients had the operation on their right side and 29 (59.2\%) had the operation in the left side. The mean perioperative hemoglobin for the patients was $9.02+2.02$. The minimum hemoglobin was 6 and the maximum hemoglobin was 14 . 


\section{Cureus}

Demographic Characteristics

American Society of Anesthesiologist Class

2

3

Operated Side

Right

Left

Implant Type Used

Cemented

Uncemented

1

48

2.00

98.00

Perioperative Hemoglobin

Mean

Standard Deviation

Minimum

6

Maximum

TABLE 2: Operative profiles of sickle cell disease patients who underwent total hip arthroplasty $(n=49)$

Figure 1 displays the avascular necrosis staging of the SCD patients who underwent THA using the Steinberg classification system. Four patients (8.2\%) had stage 2 (i.e. cystic and sclerotic radiographic changes), three (6.1\%) had stage 3 (i.e. subchondral lucency or crescent sign), 16 (32.7\%) had stage 4 (flattening of the femoral head), 10 (20.4\%) had stage 5 (i.e. joint space narrowing with or without acetabular involvement), and 16 (32.7\%) had stage 6 (i.e. advanced degenerative changes).

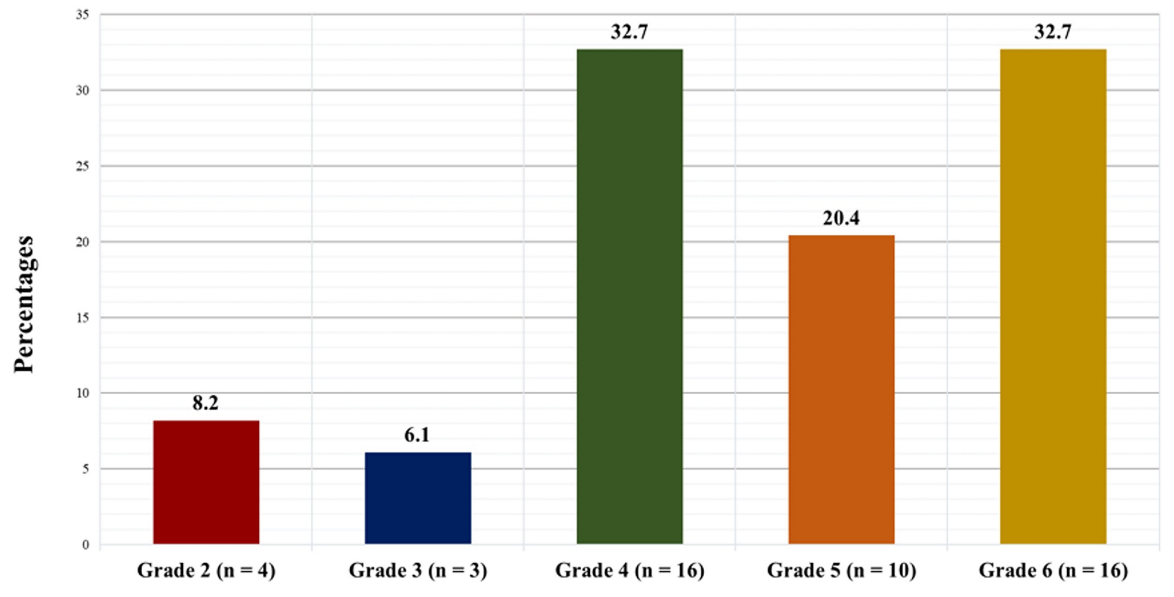

FIGURE 1: Avascular necrosis staging using the Steinberg classification system

Figure 2 displays the radiological proximal femoral morphology using the Dorr classification of the SCD 


\section{Cureus}

patients who underwent THA: 37 (75.5\%) of the patients had type A morphology, and 12 (24.5\%) had type B morphology.

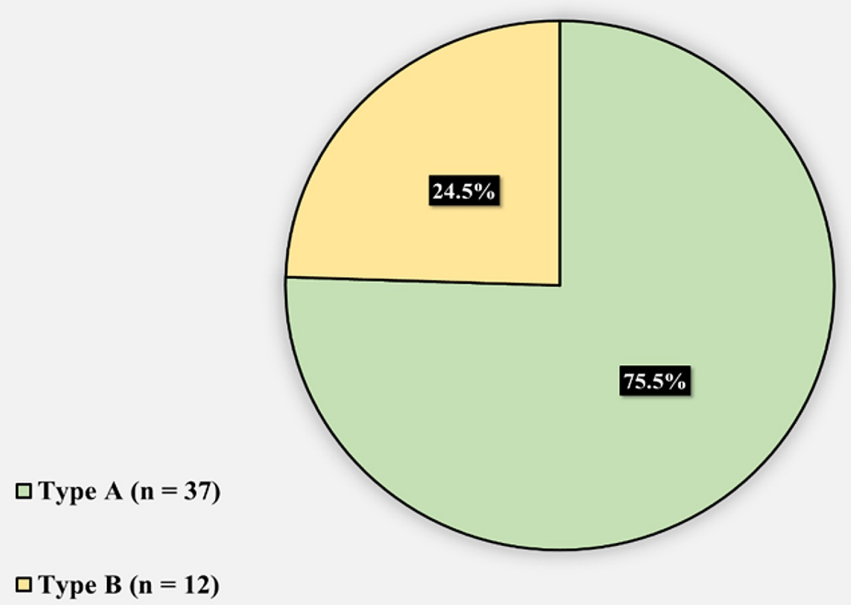

FIGURE 2: Radiological proximal femoral morphology using the Dorr classification

Figure 3 demonstrates the prevalence of intra-operative periprosthetic femoral fractures in SCD patients who underwent THA: 10 (20.4\%) had an intra-operative periprosthetic femoral fracture and 39 (79.6\%) did not.

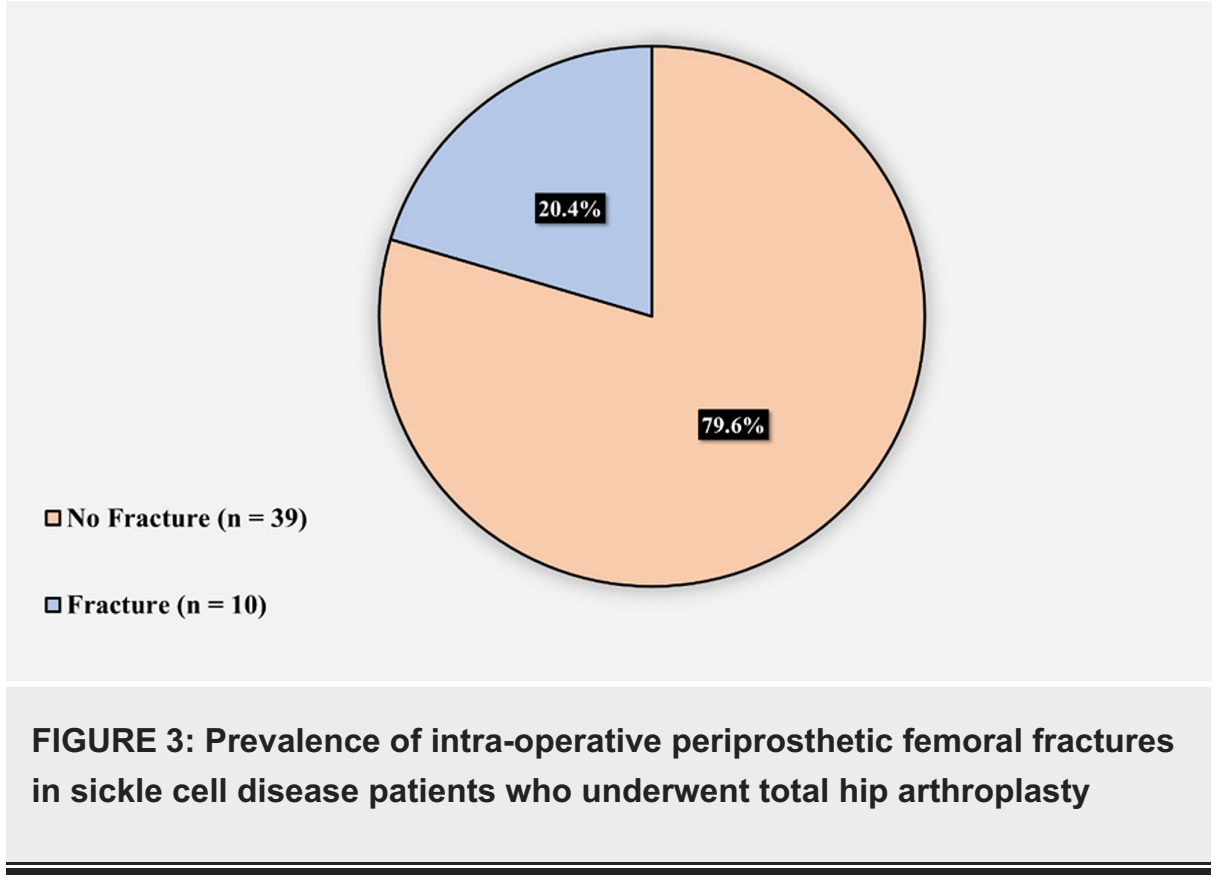

Figure 4 demonstrates the periprosthetic femoral fracture Vancouver system classes in SCD patients who underwent THA: 9 (90\%) had a class A femoral fracture and 1 (10\%) had a class B1 femoral fracture. 


\section{Cureus}

$\square$ Class A (n=9)

$\square$ Class $B 1(n=1)$

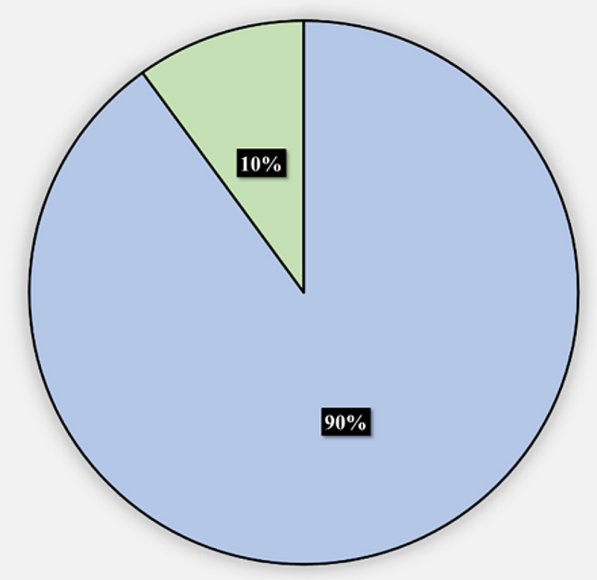

FIGURE 4: Periprosthetic femoral fracture according to the Vancouver classification system

Table 3 represents the association between the incidence of intraoperative femoral fracture during THA in SCD patients and the demographic variables and operative profiles. There was a significant association between the operated site and the incidence of intraoperative femoral fracture $(p=0.035)$, and a higher rate of fracture was observed in patients operated on the right side compared to patients operated on the left side (35\% vs. 10.3\%). No significant association was found between the incidence of intraoperative femoral fracture during THA in SCD patients and the demographic variables or the other operative profile characteristics. 


\section{Cureus}

\begin{tabular}{|c|c|c|c|}
\hline \multirow{2}{*}{ Demographic Characteristics } & \multicolumn{2}{|c|}{ Incidence of Femoral Intraoperative Fracture During Total Hip Arthroplasty } & \multirow[b]{2}{*}{ P-Value } \\
\hline & No & Yes & \\
\hline \multicolumn{4}{|l|}{ Gender } \\
\hline Male & $12(75 \%)$ & $4(25 \%)$ & \multirow[t]{2}{*}{0.579} \\
\hline Female & $27(81.8 \%)$ & $6(18.2 \%)$ & \\
\hline Age (mean, SD) & $41.41+11.85$ & $44.10+8.65$ & 0.505 \\
\hline \multicolumn{4}{|l|}{ BMI } \\
\hline Underweight & $3(100 \%)$ & $0(0 \%)$ & \multirow{4}{*}{0.612} \\
\hline Normal BMI & $21(80.8 \%)$ & $5(19.2 \%)$ & \\
\hline Overweight & $9(69.2 \%)$ & $4(30.8 \%)$ & \\
\hline Class 1 obesity & $6(85.7 \%)$ & $1(14.3 \%)$ & \\
\hline Perioperative Hemoglobin (mean, SD) & $9.14+2.08$ & $8.54+1.76$ & \\
\hline \multicolumn{3}{|c|}{ American Society of Anesthesiologist Class } & \multirow{4}{*}{0.237} \\
\hline 1 & $3(100 \%)$ & $0(0 \%)$ & \\
\hline 2 & 20 (71.4\%) & $8(28.6 \%)$ & \\
\hline 3 & 16 (88.9\%) & 2 (11.1\%) & \\
\hline \multicolumn{4}{|l|}{ Operated Side } \\
\hline Right & $13(65 \%)$ & $7(35 \%)$ & \multirow[t]{2}{*}{$0.035^{\star}$} \\
\hline Left & $26(89.7 \%)$ & $3(10.3 \%)$ & \\
\hline \multicolumn{3}{|l|}{ Implant Type Used } & \multirow{3}{*}{0.609} \\
\hline Cemented & $1(100 \%)$ & $0(0 \%)$ & \\
\hline Uncemented & 38 (79.2\%) & $10(20.8 \%)$ & \\
\hline \multicolumn{3}{|l|}{ Avascular Necrosis Stage } & \multirow{6}{*}{0.837} \\
\hline 2 & $3(75 \%)$ & $1(25 \%)$ & \\
\hline 3 & $3(100 \%)$ & $0(0 \%)$ & \\
\hline 4 & 13 (81.3\%) & 3 (18.8\%) & \\
\hline 5 & $7(70 \%)$ & $3(30 \%)$ & \\
\hline 6 & $13(81.3 \%)$ & $3(18.8 \%)$ & \\
\hline
\end{tabular}

TABLE 3: Association between the incidence of intraoperative femoral fracture during total hip arthroplasty in sickle cell disease patients and the demographic variables and operative profiles

\section{Discussion}

Patients with avascular necrosis related to SCD can be severely disabled by the status of their hip. Total hip arthroplasty remains the only surgical option for some of these patients given severe degenerative changes after collapse. Total hip arthroplasty for SCD patients can be a challenging procedure; they demonstrate high percentages of medical, intraoperative, and postoperative complications and implant failure. Because the need for THA following avascular necrosis in the Eastern Province of Saudi Arabia is high, the subsequent risk of periprosthetic fracture is common; therefore, it was crucial to conduct this study.

The study determined that $32.7 \%$ of the patients who underwent THA at King Fahad Hospital Hofuf had advanced degenerative changes due to avascular necrosis. Among these patients, 20.4\% had intraoperative periprosthetic femoral fractures, $90 \%$ had class A femoral fractures, and 10\% had class B1 femoral fractures. With our skilled SCD team and the multidisciplinary management of patients undergoing THA, our rates of 
intraoperative fracture are equal or close to those observed in the literature. However, our data did not indicate complete THA safety in this population. By comparing the outcomes of our patients with those reported in the literature, our results can help patients and orthopedic surgeons to better understand the relative risks and outcomes of this procedure in this population. However, even with adequate perioperative hydration, oxygen supply, warming, exclusion of infection sites, and conservative transfusion protocols to maintain appropriate $\mathrm{Hb}$ level, orthopedic surgeons must be prepared to deal with the high rate of intraoperative fracture.

The metaphyseal femoral morphology is typically distorted in SCD patients, characterized by thin trabeculae and cortices, medullary hyperplasia, low bone density, and patchy areas of bone sclerosis that can obliterate the femoral canal; therefore, the subsequent risk of periprosthetic fracture is greater [24]. The literature review determined that Dorr type $\mathrm{C}$ has a higher risk of fracture. In our study, 37 (75.5\%) patients had type A, 12 (24.5\%) had type B. We recommend further studies to investigate this aspect. For example, Kenanidis et al. [24] recommended facilitating femoral reaming by introducing a $4.5-\mathrm{mm}$ drill bit or a high-speed burr under an image intensifier and preparing the femoral bone to accommodate flexible intramedullary guide wires to perform cannulate intramedullary reaming.

Intraoperative periprosthetic fractures are becoming more common given the increased prevalence of revision THA and the use of uncemented fixation [25]. Many other studies have also demonstrated that uncemented implants are more associated with intraoperative complications, and almost all reported intraoperative femoral fractures were associated with uncemented THA [26]. In our study, 48 patients had an uncemented implant, and only one had cemented. Different implant options, such as short or long stems, revision options, and plates for periprosthetic fractures must be available to deal with intraoperative complications.

We found a significant association between the operated site and the incidence of intraoperative femoral fracture: a higher rate of fracture was observed in patients operated on the right side compared to the left side. Maybe, this can be explained as all of our surgeons and assistants are right-handed, so they will have better control when operating on the left side as compared to the right side. As far as we know, this is the first study to find this, so we recommend further studies to investigate this aspect. However, by comparing the gender, age, BMI, and ASA classification of our patients with those reported in the literature, we found no significant association between the incidence of intraoperative femoral fracture during THA in SCD patients.

The main limitation of this study was the low number of participants due to the small population group compared to the general population; therefore, we recommend further multicenter studies to be conducted in Saudi Arabia's, Eastern Province. This was a retrospective study, so the analyses of the surgical complications were limited by a lack of clinical detail. Additionally, the published studies we reviewed contained low levels of evidence: the majority were non-randomized retrospective case series reporting outcomes over the last 40-50 years [26-30].

\section{Conclusions}

The prevalence of periprosthetic intraoperative fracture among SCD patients at King Fahad Hospital Hofuf was $20.4 \%$ during the study period, January 2015 to September 2020. Even with adequate perioperative management, orthopedic surgeons must be prepared to deal with the high rate of intraoperative fracture. Of the patients, $90 \%$ had a class A femoral fracture, and 10\% had a class B1 femoral fracture. No significant association was found between the incidence of intraoperative femoral fracture in SCD patients and the demographic variables or the operative profile characteristics. However, a significantly higher rate of fracture was observed in patients operated on the right side compared to patients operated on the left side. Finally, we recommend a future multicenter study to be conducted in Saudi Arabia's, Eastern Province.

\section{Additional Information \\ Disclosures}

Human subjects: All authors have confirmed that this study did not involve human participants or tissue. Animal subjects: All authors have confirmed that this study did not involve animal subjects or tissue. Conflicts of interest: In compliance with the ICMJE uniform disclosure form, all authors declare the following: Payment/services info: All authors have declared that no financial support was received from any organization for the submitted work. Financial relationships: All authors have declared that they have no financial relationships at present or within the previous three years with any organizations that might have an interest in the submitted work. Other relationships: All authors have declared that there are no other relationships or activities that could appear to have influenced the submitted work.

\section{References}

1. Brodén C, Mukka S, Muren O, et al.: High risk of early periprosthetic fractures after primary hip arthroplasty in elderly patients using a cemented, tapered, polished stem. Acta Orthop. 2014, 86:169-174.

$10.3109 / 17453674.2014 .971388$ 
2. Monzón D, Iserson K, Jauregui J, Musso C, Piccaluga F, Buttaro M: Total hip arthroplasty for hip fractures . Geriatr Orthop Surg Rehabil. 2014, 5:3-8. 10.1177/2151458514520700

3. Cook R, Jenkins P, Walmsley P, Patton J, Robinson C: Risk factors for periprosthetic fractures of the hip: a survivorship analysis. Clin Orthop Relat Res. 2008, 466:1652-1656. 10.1007/s11999-008-0289-1

4. Della Rocca G, Leung K, Pape, H: Periprosthetic fractures: epidemiology and future projections. J Orthop Trauma. 2011, 25:66-70. 10.11138/ccmbm/2017.14.1.189

5. Calori G, d'Imporzano M, Tagliabue L: Periprosthetic fractures of the femur after total hip arthroplasty . Injury Extra. 2009, 40:209. 10.1016/j.injury.2009.06.236

6. King S, Lamb J, Cage E, et al.: Periprosthetic femoral fractures following total hip and total knee arthroplasty. Maturitas. 2018, 117:1-5. 10.1016/j.maturitas.2018.08.010

7. Alotaibi M: Sickle cell disease in Saudi Arabia: a challenge or not . J Epidemiol Glob Health. 2017, 7:99. 10.1016/j.jegh.2016.12.006

8. Katz J, Wright E, Polaris J, Harris M, Losina E: Prevalence and risk factors for periprosthetic fracture in older recipients of total hip replacement: a cohort study. BMC Musculoskelet Disord. 2014, 15:10.1186/14712474-15-168

9. Steinberg M, Hayken G, Steinberg D: A quantitative system for staging avascular necrosis . J Bone Joint Surg Br. 1995, 77:34-41. 10.1302/0301-620X.77B1.7822393

10. Ahmed A, Abdelkarim A, Alzahrani T, Alshahrani S, Alghamdi A, Leslom A: Incidence of avascular necrosis of the head of the femur among sickle cell patients in Albaha region, Kingdom of Saudi Arabia. Int J Med Dev Ctries. 2020, 469-472.

11. Gruson K, Aharonoff G, Egol K, Zuckerman J, Koval K: The relationship between admission hemoglobin level and outcome after hip fracture. J Orthop Trauma. 2002, 16:39-44. 10.1097/00005131-200201000-00009

12. Ilyas I, Alrumaih H, Rabbani S: Noncemented total hip arthroplasty in sickle-cell disease: long-term results . J Arthroplasty. 2018, 33:477-481. 10.1016/j.arth.2017.09.010

13. Masri B, Meek R, Duncan C: Periprosthetic fractures evaluation and treatment. Clin Orthop Relat Res. 2004, 420:80-95. 10.1097/00003086-200403000-00012

14. Zhu Y, Chen W, Sun T, Zhang X, Liu S, Zhang Y: Risk factors for the periprosthetic fracture after total hip arthroplasty: a systematic review and meta-analysis. Scand J Surg. 2014, 104:139-145. 10.1177/1457496914543979

15. McLaughlin J, Lee K: Long-term results of uncemented total hip arthroplasty with the Taperloc femoral component in patients with Dorr type C proximal femoral morphology. Bone Joint J. 2016, 98:595-600. 10.1302/0301-620x.98b5.35816

16. Kim J, Jeong H, Lee S, Kim H, Yoo J: Does proximally coated single-wedge cementless stem work well in Dorr type C femurs? minimum 10-year followup. Indian J Orthop. 2019, 53:94-101.

17. Parker M, Pryor G, Gurusamy K: Cemented versus uncemented hemiarthroplasty for intracapsular hip fractures. J Bone Joint Surg Br. 2010, 92:116-122. 10.1302/0301-620X.92B1.22753

18. Azegami S, Gurusamy K, Parker M: Cemented versus uncemented hemiarthroplasty for hip fractures: a systematic review of randomised controlled trials. HIP International. 2011, 21:509-517. 10.5301/HIP.2011.8640

19. Deng Y, Kieser D, Wyatt M.: Risk factors for periprosthetic femoral fractures around total hip arthroplasty: a systematic review and meta-analysis. ANZ J Surg. 2019, 10.1111/ans.15473

20. Radunović A, Vulović M, Aksić M, Radunović O, Matić A: Simultaneous bilateral joint arthroplasties in treatment of osteoarthritis. Osteoarthritis. Amarasekera HW (ed): IntechOpen, London; 2020. 10.5772/intechopen.93147

21. Terminology - avascular necrosis-osteonecrosis education and resources . (2020). Accessed: 23 October 2020: https://avascularnecrosiseducation.com/category/terminology/.

22. Nash W, Harris A: The Dorr type and cortical thickness index of the proximal femur for predicting perioperative complications during hemiarthroplasty. J Orthop Surg. 2014, 22:92-95. $10.1177 / 230949901402200123$

23. Of periprosthetic fractures about/below total hip arthroplasty . (2020). Accessed: 23 October 2020: https://plasticsurgerykey.com/of-periprosthetic-fractures-about-below-total-hip-arthroplasty/ .

24. Kenanidis E, Kapriniotis K, Anagnostis P.: Total hip arthroplasty in sickle cell disease: a systematic review . EFORT Open Rev. 2020, 5:180-188. 10.1302/2058-5241.5.190038

25. Davidson D, Pike J, Garbuz D.: Intraoperative periprosthetic fractures during total hip arthroplasty. J Bone Joint Surg Am. 2008, 90:2000-2012. 10.2106/jbjs.h.00331

26. Bishop A, Roberson J, Eckman J.: Total hip arthroplasty in patients who have sickle-cell hemoglobinopathy . J Bone Joint Surg Am. 1988, 70:853-855. 10.2106/00004623-198870060-00009

27. Sharma M, Gulati Y, Bharti B, Bahl V, Bohra I, Goswani A: Short term results of cementless total hip arthroplasty in sicklers. Indian J Orthop. 2015, 49:447.

28. Hickman J, Lachiewicz P: Results and complications of total hip arthroplasties in patients with sickle-cell hemoglobinopathies. J Arthroplasty. 1997, 12:420-425. 10.1016/s0883-5403(97)90198-4

29. Hanker G, Amstutz H: Osteonecrosis of the hip in the sickle-cell diseases: treatment and complications . J Bone Joint Surg Am. 1988, 70:499-506. 10.2106/00004623-198870040-00004

30. Acurio M, Friedman R: Hip arthroplasty in patients with sickle-cell haemoglobinopathy . J Bone Joint Surg Br. 1992, 74:367-371. 10.1302/0301-620X.74B3.1587879 